\title{
INTERACTIVE FACTORY SCHEDULING USING DISCRETE EVENT SIMULATION
}

\author{
Dr. T. A. Norman \\ Van B. Norman \\ AutoSimulations, Inc. \\ P.O. Box 307 \\ Bountiful, UT 84010
}

\begin{abstract}
Interactive simulation based scheduling offers new and powerful capabilities to the manufacturing manager. The effectiveness of the technique is dependent on the completeness of the data base, the ease of use and the ability of the model to accurately reflect actual manufacturing situations. The use of these techniques allows floor level managers to spend their time on personnel management, not as high paid bookkeepers.
\end{abstract}

\section{INTRODUCTION}

Scheduling involves the sequencing of work at manufacturing centers within the conficting constraints of inventory minimization, equipment and operator utilization, and order delivery dates. Simulation is a proven engineering technique in the manufacturing environment because of its powerful ability to imitate the real world with minimal underlying assumptions. Simulation based scheduling will become a useful tool in manufacturing because of recent advances in computing equipment, artificial intelligence software and shop floor data collection systems.

The manufacturing environment continues to change. Every manufacturer is being driven to reduce operating costs and improve quality by an increasingly competitive world wide economy. The extra costs incurred by excess inventory, long delivery schedules and low utilization of resources cannot be maintained. This environment has greatly increased the operational problems for manufacturing schedulers. All the fat is being removed from manufacturing facilities. There is no more excess inventory or low utilized machines. Schedules must be short and accurate. And yet the minute by minute operation is very dynamic. Operators get sick and machines fail. Schedulers need a tool that allows them to investigate the effects of management decisions (scheduling rules) before they are made. Simulation based scheduling is that tool.

\section{CURRENT APPROACHES TO SCHEDULING}

Production scheduling requires the balancing of many conflicting requirements. For example: To reduce the delivery time between the customer order and the delivery date may require an increase in work-in-process inventory or machine capacity. Increasing machine utilization may require an increase in work-in-process inventory and delivery times. Three techniques for scheduling are commonly used. Either an increase in inventory or a decrease in machine utilization will increase costs; while an increase in delivery times will result in lost sales.

\subsection{Order Launch and Expedite}

Order launch and expedite is the most common method of scheduling--if it may be called scheduling. Many facilities release customer orders to the plant as they are received. The order of processing is determined by due dates and "hot sheets." Expediters dynamically move between work centers attempting to get their hot jobs started. There is little ability to predict the actual completion date of any job.

\subsection{Local Rules}

In some facilities a machine operator or first line supervisor may select the next job to be processed. The rule for selection is not based upon any global understanding of the production plan but upon the criteria by which the individual is judged. If someone is judged by conformance to standard hours; then the job selected will be the one that offers the best ratio between actual and standard hours.

Visual scheduling may be done with control boards, but these offer little ability to investigate alternatives and are difficult to modify when requirements change or a machine breaks down. Analytical techniques such as shortest processing time or weighted shortest processing time can produce good schedules but they have difficulty dealing with real world complexities such as rework, batching at heat treat and a few jobs that actually have a high priority.

\subsection{Computer Based Scheduling}

Recently, some companies have used computer based tools that identify the bottle neck operation and then attempt to work backward and forward to create operational schedules. This technique is computationally intensive and has not been used on an hour by hour basis where the manufacturing environment is very dynamic.

\section{SIMULATION BASED SCHEDULING}

Simulation has long been a powerful tool for the analysis of the complexities found in manufacturing systerns. Simulation programmers have constructed models to test scheduling rules under different operating conditions. These models require skilled individuals to construct. A tool that is simulation based but allows the scheduler to describe the manufacturing environment is an improvement in cost and ease of use. Simulation based scheduling may be used either long term as a capacity planning tool or short term as an interactive scheduler.

\subsection{Capacity Planning}

Designers of flexible manufacturing systems are beginning to realize that the performance of their systems is greatly affected by the scheduling rules in operation. Many advanced manufacturing systems cannot be simulated correctly without knowing the schedule that drives the system. Simulation based capacity planning studies are more accurate because the simulation model is an accurate representation of the facility and shows the interaction of scheduling rules, inventory levels, and resource availability. 


\section{T. A. Norman and V. B. Norman}

\subsection{Interactive Scheduling}

Manufacturing systems are dynamic with many random events; machine failures, operator availability, process yields, rework rates, etc. A simulation tool that accurately represents a manufacturing facility gives production schedulers the ability to easily investigate alternative plans. The simulation based scheduler must have the following operating characteristics to be an effective tool.

3.2.1. Accurate Data Base. Many companies have computer data bases of their process plans (part routings). Experience has shown that what actually happens on the shop floor is frequently different than what the process planner intended and is only known by the floor level personnel.

On line shop floor data collection systems are a requirement for dynamic scheduling. When labor and production order reporting are accomplished over night, the simulation based scheduler may produce a schedule for the next day's operations.

3.2.2. Execution Speed. The simulation based scheduler must be able to simulate the next shift in a few minutes so that the user has sufficient time to investigate alternatives.

3.2.3. Ease of Use. The scheduler must allow the user to describe the system in statements that make sense to the user and have output that can be looked at in a number of graphical formats.

\section{REQUIRED FEATURES}

A simulation based factory scheduler must have certain functional features in order to accurately model manufacturing facilities. These features can be grouped into three areas: 1) Resources, 2) Parts and 3) Rules. The following discussion gives examples of the required features and defines the appropriate level of detail for feature definition.

\subsection{Resources}

The resources in a model of a manufacturing system are the constraining elements. They include : 1) processing machines, 2) operators, 3) tooling and fixtures 4) material storage areas and 5) material handling equipment.

4.1.1. Machines. Machines in the factory may belong to a family of similar machines for scheduling purposes. Additional required characteristics include:

Location in the Factory

Local Input and Output Storage Space

Available Operating Schedule

Setup Requirements

Processing Requirements

MTBF and MTTR Data

Machine Dependent Scheduling Rules

4.1.2. Operators. Operators may belong to skill levels or job classifications and must have the following characteristics:

Number Available

Schedule of Availability

4.1.3. Tooling and Fixtures. Tooling and fixtures are frequently the constraining resource and must have the following characteristics:

Number Availablè

Cleaning and Inspection Procedures
4.1.4. Material Storage Areas. Material storage areas must have the following characteristics:

Location

Space Available

4.1.5. Material Handling Equipment. Material handling equipment must have the following characteristics:

Number Available

Movement Capacity

Movement Restrictions

Velocity

\subsection{Parts}

The definition of parts in the model must include two elements: 1) the processing steps required to make each part and 2) the production orders for the parts.

4.2.1. Processing Plans. Processing plans for parts define the processing steps each part must follow. They must allow for the following situations:

Alternate and nonsequential routings
Rework
Assembly operations
Batching and consolidation operations
Material life requirements

4.2.2. Production Orders. Production order data includes the individual orders with due dates and production quantity requirements. For orders in-process the online data collection system must report the status of every order.

\subsection{Rules}

The operating rules selected for the facility will be evaluated by the simulator. The rules should define the following: 1) operating schedules, 2) load movement rules, 3) intraorder sequencing rules and 4) machine scheduling rules.

4.3.1. Operating Schedules. The work schedules for machines and operator classes tell which days and shifts they are available for use and, in the case of operator classes, how many operators of the class are available. For each shift of each day of the simulation period each machine should be specified as either on or off shift. The numbers of operators of each class for each shift should likewise be specified.

4.3.2. Lad Movement Rules. Load movement rules determine where and when loads are moved after they complete an operation. The load may be kept at the current operation, moved to the next operation or placed in a storage area.

4.3.3. IntraOrder Sequencing Rules. For muitiple load orders it should be possible to treat the loads almost independently, to require that the loads pass through their processing sequence in "lock step", or to require some compromise between these two extremes.

4.3.4. Task Selection Rules. When a machine becomes free and multiple loads are waiting to be processed by a machine of its family then it is necessary to choose one of the loads as the next task for the machine. Task selection rules are the rules followed by machines in making such choices.

A task selection rule is a sequence of criteria which are used one by one to reduce the number of possible choices to one. 


\section{CONCLUSION}

Interactive simulation based scheduling offers new and powerful capabilities to the manufacturing manager. The effectiveness of the technique is dependent on the completeness of the data base, the ease of use and the ability of the model to accurately reflect actual manufacturing situations. The use of the techniques allows floor level managers to spend their time on personnel management, not as high paid bookkeepers.

\section{AUTHOR'S BIOGRAPHIES}

THEODORE A. NORMAN, vice president of AutoSimulations, Inc., was chairman of the Computer Sciences Department at Brigham Young University. He received a B.S. in Mathematics from the University of Utah (1962), an M.S. in Information Science from Washington State University (1968), and a Ph.D. in Information Science at Washington State Univeristy (1970). He was a Systems Engineer for IBM and a consultant in simulation and controls design. $\mathrm{He}$ is currently involved in the development of new scheduling tools for manufacturing applications.

Theodore A. Norman

AutoSimulations. Inc.

P.O. Box 307

Bountiful, UT 84010

(801) 298-1398

VAN B. NORMAN, vice president of AutoSimulations, Inc., received a B.S. in Mathematics at the University of Utah in 1969. He was a senior systems analyst for Eaton-Kenway Co. and the Utah Board of Education, for which he developed a state-wide computer network. At AutoSimulations, he supervises all simulation modeling and graphics development.

Van B. Norman

AutoSimulations, Inc.

P.O. Box 307

Bountiful, UT 84010

(801) 298-1398 